\title{
A NUMERICAL AND EXPERIMENTAL APROACH OF STRESS WAVES PROPAGATION IN SHORT TRONCONICAL BARS UNDER AXIAL IMPACT
}

\author{
Nicolae ILIESCU*, Vasile NĂSTĂSESCU**, Ghiță BÂRSAN*** \\ *6"Politehnica" University, Bucharest, Romania \\ **Military Technical Academy, Bucharest, Romania \\ ***“Nicolae Bălcescu” Land Forces Academy, Sibiu, Romania \\ nviliescu@yahoo.com
}

\begin{abstract}
In the first part of the paper, using the numerical simulations with FEM and the results of some investigations made with different experimental techniques, a calculation methodology was developed for the study of the stress waves propagation in the short tronconical bars subjected at axial impact. Because a good agreement between data obtained from numerical analysis and experimental investigations was observed, the numerical model of calculus conceived for this study was considered validated. The calculus model established was used to investigate other aspects connected of stress wave propagation in the short tronconical bars. In the second part of the paper, using established calculus model and numerical analysis with Finite Element Method the influence of bar conicity on stress wave propagation and on stress distribution in different cross sections of the bar was analyzed.
\end{abstract}

\section{Keywords: strain, stress wave, finite elements, dynamic photoelasticity}

\section{Finite Element Model}

Numerical analysis was carried out considering a truncated cone with apex angle of $20^{\circ}$. The three dimensional calculus model was meshed with solid and tetrahedral elements, with variable dimensions, as it can see in the Figure 2.

For a fidelity reproducing of the experiment, a metallic cylinder of $12.7 \mathrm{~mm}$ diameter was placed on the head of the truncated cone.

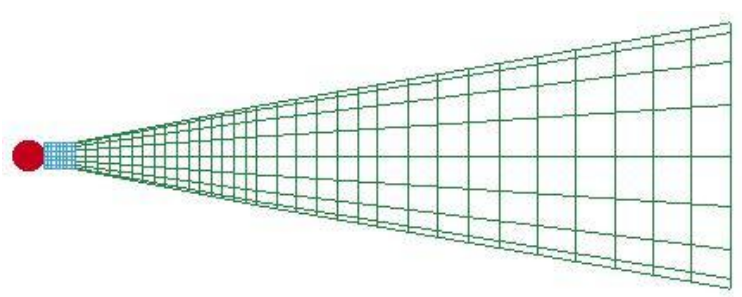

Fig. 1. Finite element model
The base of the model was clamped. The stress longitudinal wave was generated by shutting the small end of the bar with a 12.7 $\mathrm{mm}$ diameter metallic ball with $29.2 \mathrm{~m} / \mathrm{s}$ velocity. The numerical simulations regarding the wave stress propagation, along the truncated cone were done using the LS-Dyna program.

\section{Numerical Analysis Results}

In order to highlight the propagation process of the stress wave, along the model and to determine the stress distribution in different cross sections of the bar, a special post processor was used.

A three dimensional image of the wave front, cached along tronconical bar, at three successive intervals of time after impact is illustrated in Figure 2. Intersection of the three dimensional image of the wave front with longitudinal plane of the model, at

DOI: $10.1515 / \mathrm{kbo}-2018-0144$

(C) 2015. This work is licensed under the Creative Commons Attribution-NonCommercial-NoDerivatives 3.0 License. 
these intervals of time is presented in Figure 3.

As we can see in these figures, the stresswave front is spherical, with the maximum stress at the central axis of the cone. Also, it can see that the wave front radius increases as pulse travels towards the large end of the conical model.

The stress wave front, in the longitudinal plane of the bar, at three different analysis time, is presented in Figure 4.

From these diagrams it is evident that initial pulse propagates with a great attenuation.

The attenuation of the stress level between section I and section III is about $33.4 \%$ for a length of $100 \mathrm{~mm}$. This diagram, also shows that the negative tale of the pulse develops as the wave travels down the cone.

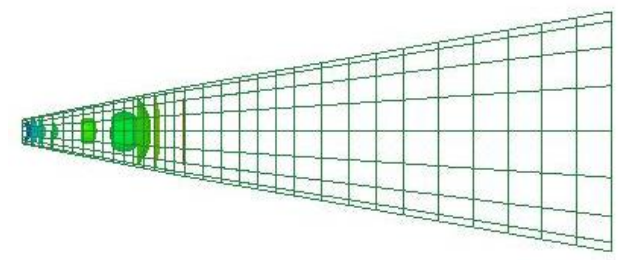

a)

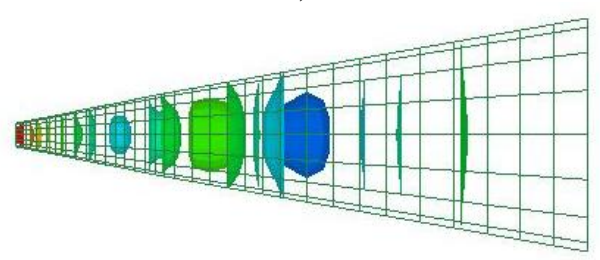

b)

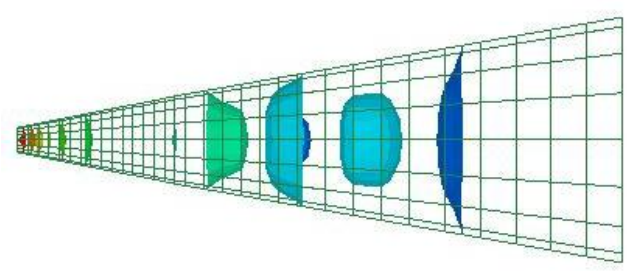

c)

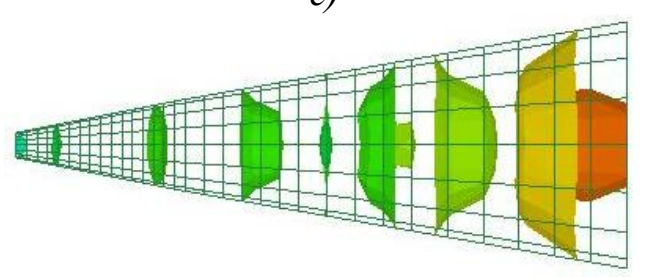

d)

Fig. 2. Surfaces of stress isovalues at different analysis time: 50, 100, 150 and 220 microseconds
A close examination of the Figures 3 and 4 shows that the stress wave front is followed by a more complex pattern of waves which seems to represent a trailing portion of the pulse.

Using numerical analysis data, the stresses $\sigma_{y}$ in the three different sections on the surface of the model, are plotted in the Figure 5. The same simulations were done for truncated cones with different apex angle.

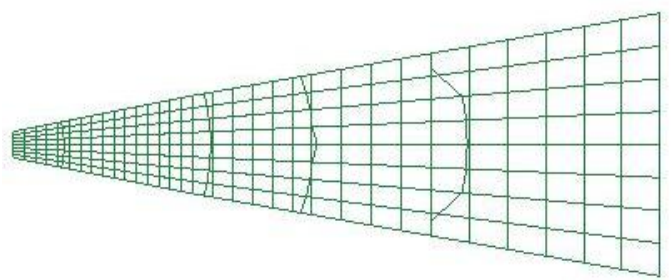

$100 \mu s$

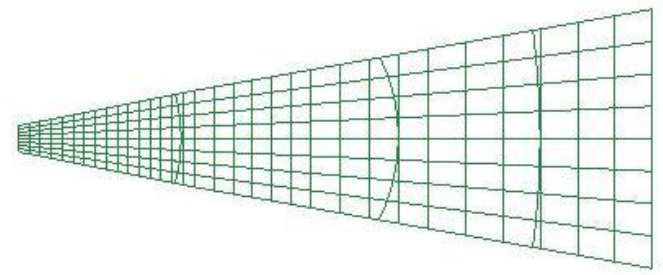

$200 \mu s$

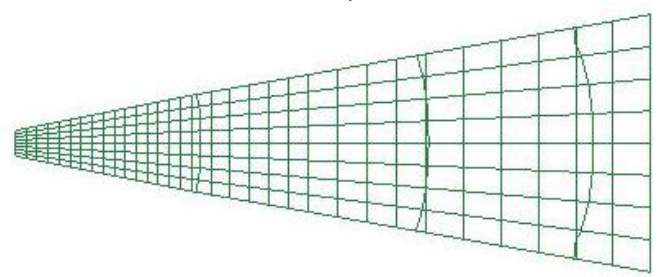

$200 \mu s$

Fig. 3. Stress wave propagation

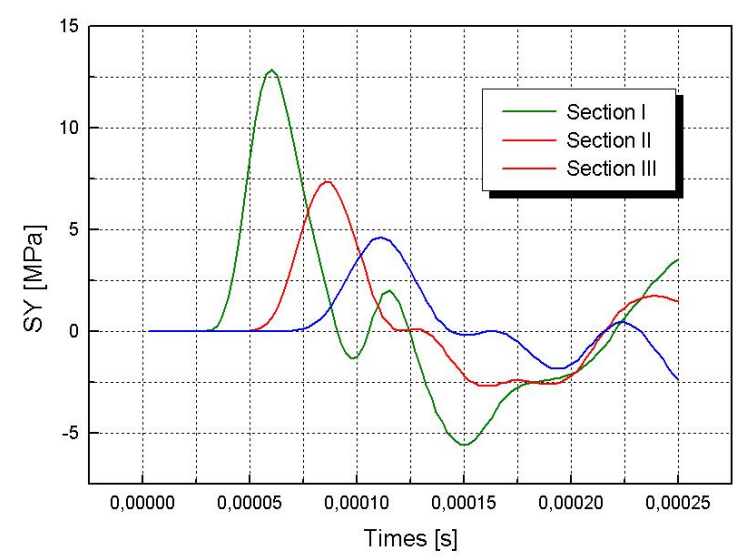

Fig. 4. Stress variation $\left(\sigma_{y}\right)$ in the three sections 
In the Figure 6, the variation curves of $\sigma_{y}$ stress vs. apex angle, for three different section $(\mathrm{A}, \mathrm{B}, \mathrm{C})$ are plotted.

These diagrams indicate that the surface stresses $\sigma_{y}$ decrease as the apex angle increase. The grater difference between values of these stresses occur for angles between 10 to 30 degrees.
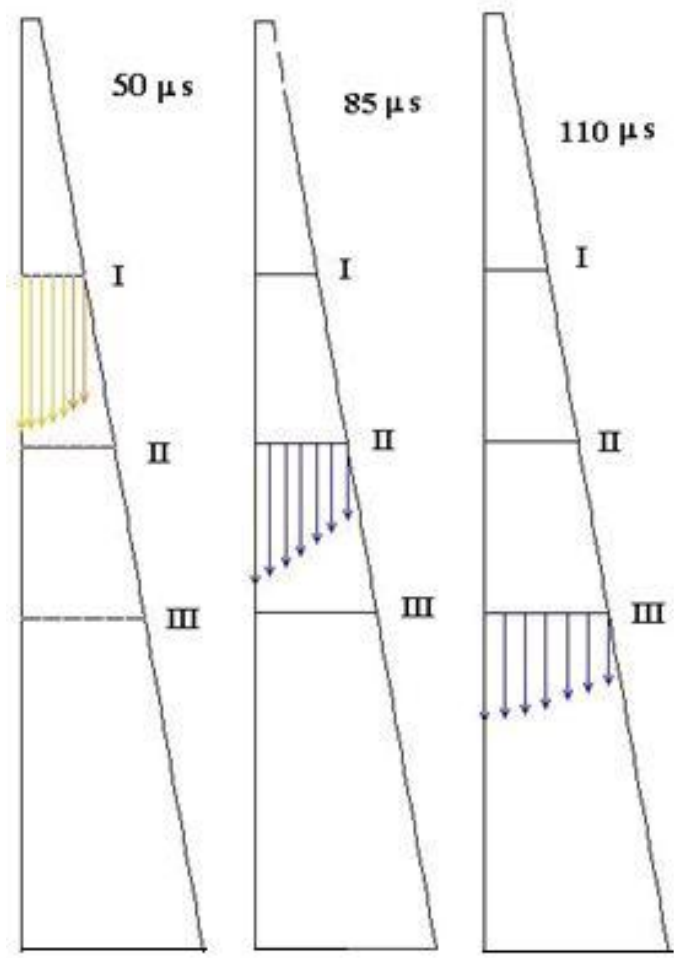

Fig. 5. The variation of the $\sigma_{y}$ stress in cross sections I, II and III

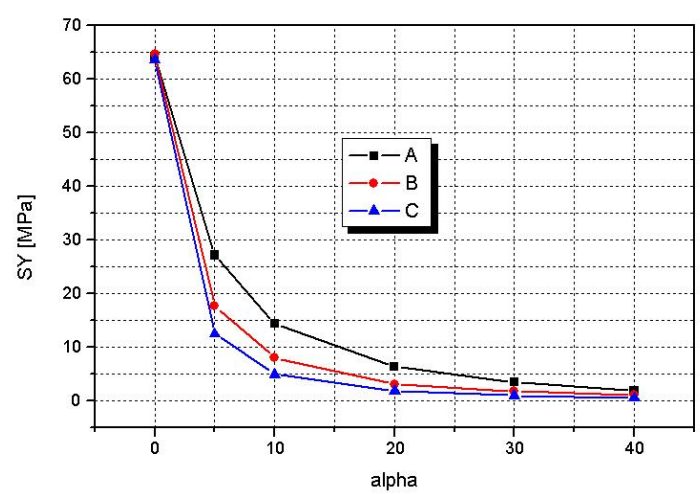

Fig. 6. Variation of SY stress vs. apex con angle

\section{Experimental Investigations}

In order to validate conceived calculus model, an experimental investigation on elastic wave propagation in a truncated cone with apex angle of $20^{\circ}$, was conducted by dynamic photoelasticity [2].

Metamorphosis of the photoelastic fringes caused by the stress wave propagation in conical bar was recorded using three different experimental techniques: photo elasticity in dynamic regime, using highspeed framing camera, straingauge technique and laser-photomultiplier system. For control purposes, the strains history along the cone surface was also recorded by strain gauge technique.

The photoelastic model tested in this investigation was a truncated cone with apex angle of $20^{\circ}$, machined of bakelite ERL-2774. Using a slight calibration bar, a preliminary dynamic calibration of the photoelastic material was done.

Such properties as dynamic modulus of elasticity $\left(E_{D}\right)$, the dynamic Poisson's ratio $\left(v_{D}\right)$, rod wave velocity $\left(c_{0}\right)$ and dynamic stress-strain optic coefficients $\left(f_{\sigma D}\right.$ and $f_{\varepsilon D}$ ) were determinated. The photoelastic model was made of ARALDIT. Two sheets of HN-32 Polaroid ( $0.25 \mathrm{~mm}$ thick) with the polarization axes oriented at $45^{\circ}$ from the cone axis, were embedded at the interfaces of the three-pieces of composite model, forming an internal polar scope. The propagation of the stress waves was also recorded with the strain gauge technique. For this purpose in three sections of the built-in polar scope, two simple electroresistive transduscers were applied (Fig.7).

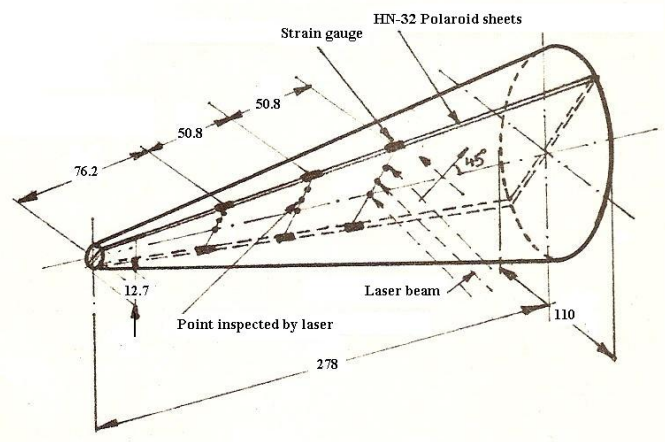

Fig. 7. Photoelastic model

To cancel the refraction of the light ray transmitted from the laser source, when it 
would encounter curve surface of the cone, the photoelastic model was placed in a mating unit of the same material but having plane external surfaces.

The specimen was separated from the mating unit by a gap of $0.1 \mathrm{~mm}$, in which it was kept a film of hallowax. The stress waves were generated in the photoelastic model by striking the small end of the model with a $12.7 \mathrm{~mm}$ diameter steel ball.

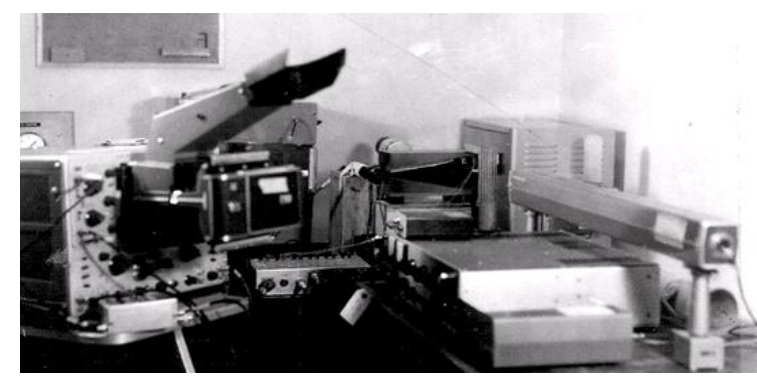

Fig. 8. Arrangement of experimental equipment

A compressed air gun operating at a maxim pressure of 1.5 bars propelled the ball. To obtain the initial velocity of the ball, a transducer of velocity was employed.

Using laser-photomultiplier system, the photoelastc fringes dependence of time was recorded.

A laser beam of $1.1 \mathrm{~mm}$ diameter was transmitted through the photoelastic model, in three points placed in sections where strain gauges were bonded on the surface of the cone, in the longitudinal plane (Fig. 7).

The output of the photomultiplier tube was recorded by a 565 Tektronics oscilloscope. In order to determine the individual values of the principal stresses in a section of the model, the oblique incidence method was employed.

For that purpose, the cone was rotated about vertical axis, so that the light to passé through the model under an angle of $65^{\circ}$ in respect to its longitudinal axis.

To obtain the additional information on the elastic wave propagation, a Bowen RC-4 high-speed framing camera was used.

The framing camera operating at $1.2 * 10^{5}$ frames per second recorded, in sequences, evolution of photoelastic fringes, along the cone, at equal intervals of time of $8.3 \mu \mathrm{s}$.

A flash properly delayed at a certain interval of time, by the master trigger system of oscilloscope, provided a good illumination to record dynamic fringe patterns.

Simultaneously with photomultiplier response corresponding to the passage of successive fringes, the strain history was recorded by BLH SR-4 strain gauges type FAE-12-S9L, placed in the three sections.
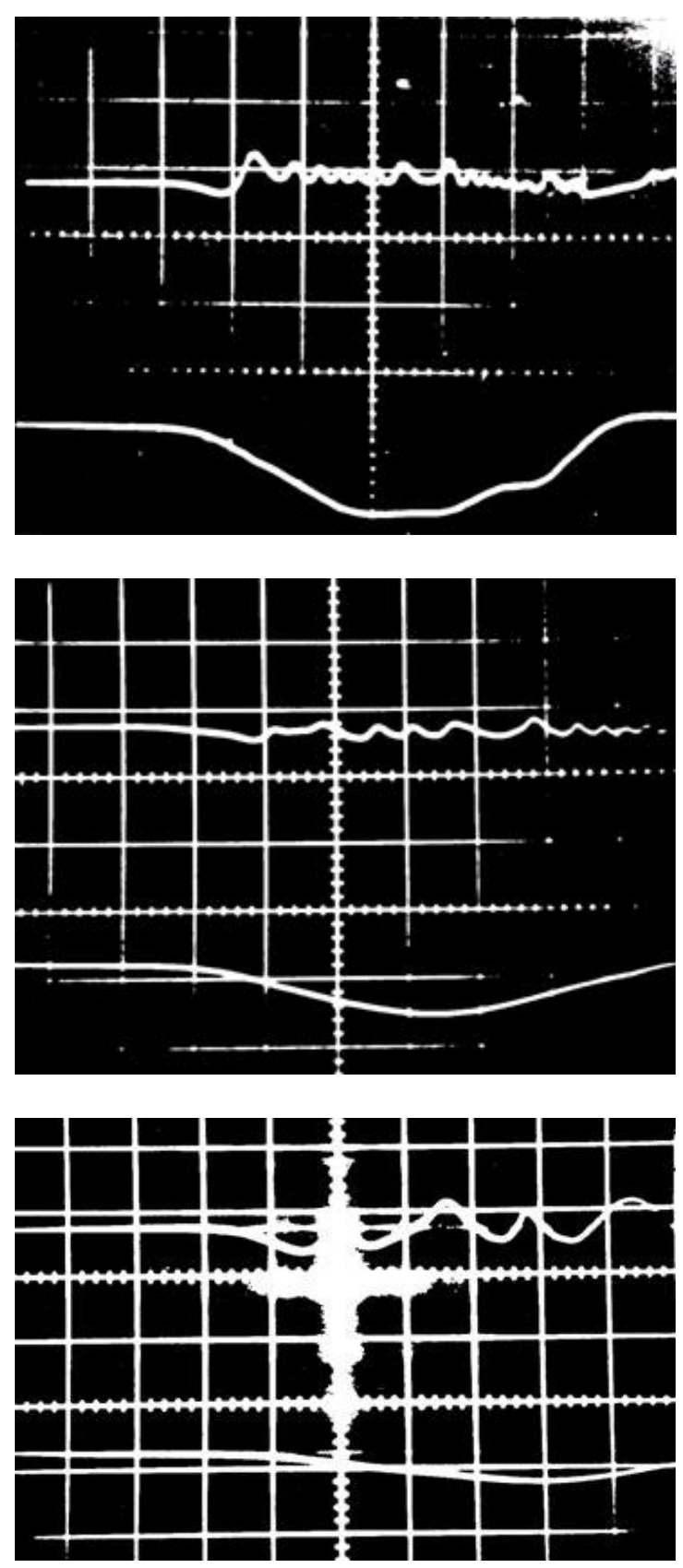

Fig. 9. Photomultiplier and strain gauge responses 
The strain gauges outputs were recorded by a 565 Tektronix oscilloscope using a 3A1 amplifier. A light-source focused on a photocell, placed normal to the ball trajectory, near the protector head, triggered the oscilloscope. The oscilloscope traces were delayed until the pulse arrived at the section of interest, using delay device of oscilloscope. The arrangement of experimental equipment employed in this investigation is presented in the Figure 8.

\section{Experimental Investigations}

As the result of the impact produced by shooting a $12.7 \mathrm{~mm}$ diameter steel ball, at the small end of the cone, a number of 6 to 7 photoelastic fringes were induced in longitudinal section of the specimen.

Photoelastic fringes metamorphosis during the stress wave propagation was recorded both by laser-photomultiplier and highspeed framing camera. In Figure 10, typical photomultiplier tube responses and corresponding strain-gauges traces (for normal incidence) are presented, for three points located on the cone axis at the three measuring stations.
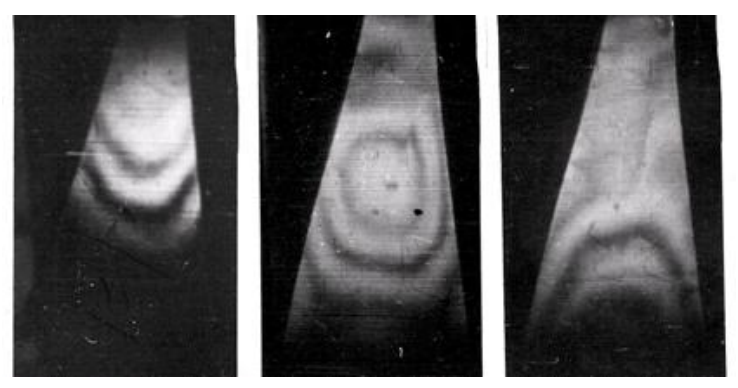

Fig. 10. The stress wave propagation

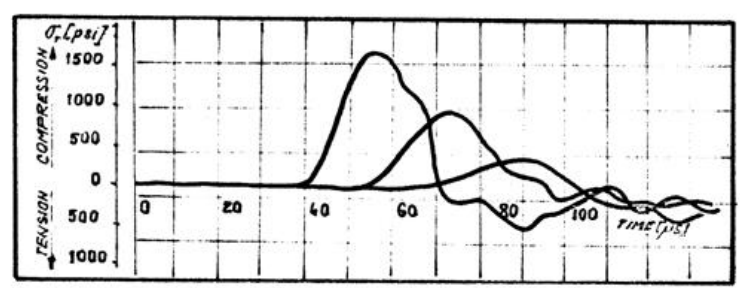

Fig. 11. Stress distribution in the three cross sections of the model

Figure 10 shows a typical sequence of frames representing fringe patterns associated with the propagation of the stress wave in longitudinal section of the cone.

First frame corresponds to a moment at about 76 microseconds after impact and others at intervals of $126,143,168$, and 176 microseconds after impact.

Inspection of records presented in Figure 9 revels a diminution of the fringe order with an increase of conical section of about $28.5 \%$ on a length of $100 \mathrm{~mm}$.

The records obtained for the other points show also that fringe order decay with increasing radius in a section. The straingauges response recorded by the three stations, presented in Figure 11, shows that initial pulse propagates with a great attenuation. The strains level attenuation between station 1 and 3 is about $35.5 \%$ for a length of $100 \mathrm{~mm}$.

A comparing of the strain gauge data and the data obtained by laser-photomultiplier analysis, indicated that dispersion of the longitudinal waves is greater at surface of the model then of the axis.

As can see in photographs presented in Figure 10 the stress-wave front is spherical in a cross section of the cone, with the maximum stress at the central axis of the cone. The photographs show also that wave-front radius increase as pulls travels toward the large end of the conical model.

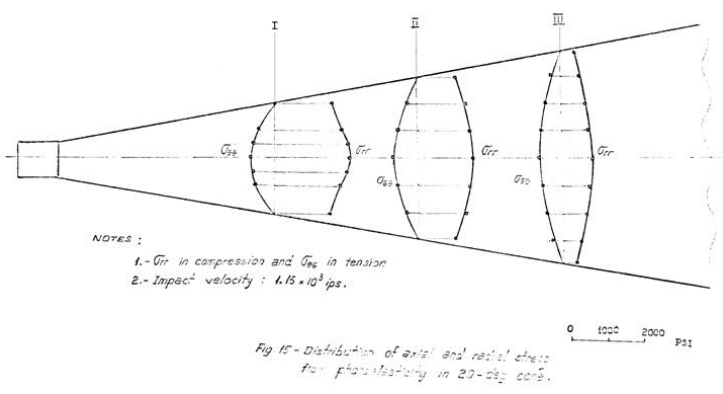

Fig. 12. Stress distribution on the cross section area and its attenuation

A close examination of these photographs shows that the stress-waves front is followed by a more complex pattern of curved fringes which seem to represent a trailing portion of the pulse. 
The separate values of the stresses $\sigma_{\mathrm{rr}}$ and $\sigma_{\theta \theta}$ were determined by oblique incidence method.

To cancel the error which can occur by applying of this method, a good repeatability of the experiment is desired.

In this way, a great number of tests were made, till the difference in fringe order of both the normal and oblique incidence was less than half a fringe.

The individual values of the stresses $\sigma_{\mathrm{rr}}$ and $\sigma_{\theta \theta}$ plotted in Figure 12 were determined considering only component $\mathrm{q}^{*} \cos ^{2} \theta$ of the stress.

The diagrams presented in Figure 11 and 12 show that the distribution of axial and radial stresses $\sigma_{\text {rr }}$ and $\sigma_{\theta \theta}$ is not uniform across a section of the cone, as the one-dimensional theory predicated.

The stresses have the maximum values at the central axis of the cone and they decrease in value with increasing distance from the impact end.

Results of this test shown that the lateral inertia has to be taken into account in the one-dimensional constitutive equation for the case of the cone with large apex angle.

\section{Discussions}

Comparing the experimental results with data provided by numerical calculus shows that in both analyses it found that:

- Initial pulse propagates with a great attenuation (Fig. 5 and Fig. 11) with increasing radius in a section.
- The stress-wave front is spherical in a cross section of the cone (Fig. 2, Fig. 4 and Fig. 10), with the maximum stress at the central axis of the cone.

- The stress-waves front is followed by a more complex pattern of curved fringes (Fig. 5 and Fig. 11), which seem to represent a trailing portion of the pulse.

- The distribution of axial and radial stresses $\sigma_{\mathrm{rr}}$ and $\sigma_{\theta \theta}$ is not uniform across a section of the cone, as the onedimensional theory predicated. The stresses have the maximum values at the central axis of the cone and they decrease in value with increasing distance from the impact end (Fig. 3 and Fig. 12).

- Dispersion of the longitudinal waves is greater at surface of the model then of the axis (Fig. 3, Fig. 4 and Fig. 12). That shows the lateral inertia has to be taken into account in the one-dimensional constitutive equation for the case of the cone with large apex angle.

\section{Conclusions}

All these aspects above mentioned, point out a good agreement between the two families of data obtained by numerical simulations and experimentally. In consequence the conceived calculus model can be used for nonlinear analysis in investigations connected with stress wave propagation in tronconical bars.

\section{References}

[1] Brillhart, L.V., and Dally, J. W. A Dynamic Photoelastic Investigation of Stress-wave Propagation in Cones,. Experimental Mechanics, 1968, pp.145-153.

[2] Iliescu, N., The Study of Stress Wave Propagation in a Tronconical Bar by Dynamic Photoelasticity, Symposium on Experimental techniques in Applied Mechanics, Bucharest, 1-3 November 1972.

[3] Kenner, V. H., and Goldsmith, W., Elastic waves in Truncated Cones, Experimental Mechanics, 8, 1968.

[4] Landon, I. W. and Quinney, I., Experiments with Hopkinson Pressure Bar, Proc.Roy.Soc. London, 1943.

[5] Năstăsescu, V., Metoda elementelor finite, Editura Academiei Tehnice Militare, Bucureşti, 1995. 